\title{
FOCAL patch for synchronized I/O, precision timing, and on-line files: On-line "reflexes" for general-purpose interpreter languages
}

\author{
T. H. NILSSON \\ University of Alberta, Edmonton, Alberta, Canada T6G 2E9
}

\begin{abstract}
To permit on-line control of experiments with an existing high-level interpreter language, this three-argument user function enables operation of the $\mathrm{I} / \mathrm{O}$ bus, clock, and timed relays to be synchronized to external events. It also supports on-line files and machine language programs in high memory fields. The patch is reprogrammable from the interpreter and takes only 28 locations of the user area to link with the remainder, which uses one page in another memory field. Some implications of this approach for on-line experimentation are discussed.
\end{abstract}

A contributing factor to the under utilization of on-line computers in psychology (Sidowski, 1976) may be represented by our experiences. As delivered, most computer systems are little more than Teletype calculators. The requisite $\mathrm{I} / \mathrm{O}$ facilities for controlling experiments (Uttal, 1969) have been either lacking or, in the case of the PDP-12, present but without the software to use them. From the more than 20 special operating programs for on-line experimentation reported in Behavior Research Methods \& Instrumentation in the past few years, it is evident that considerable programming effort must go into making computers operational in the laboratory.

Our principal concern was the familiar one discussed by Castellan (1976) of obtaining precision real-time operation of $1 / 0$ functions and of synchronizing these operations to apparatus and events external to the computer. With little programming experience or resources, our search for a solution followed the philosophy, presented by Siegel (1972), of having an existing general-purpose interactive language handle communication, organization, and calculations while we wrote short, linked patches in assembly language to handle the more specialized control functions. After going through a succession of patches to perform various functions, 1 developed a combination (FIDO) which, in spite of its simplicity, has proved adaptable to a wide variety of on-line single-experiment requirements. While most of its features have probably been developed in-house at other laboratories, a discussion of this approach may be of value to persons seeking a means of on-line programming. Though we use FIDO linked to FOCAL, it is evident that this approach can also be used to make other easy-to-use interpreter languages (like BASIC) suitable for controlling experiments. We have been using FIDO combined with FOCAL-RT

Development was supported by the National Research Council of Canada under Grants APA-145 and A0-322.
(Siegel, 1972) and FOCL-12K (Kaye, 1971) patches to FOCAL-12 on a PDP-1 2 with $12 \mathrm{~K}$ of memory. Together they give FOCAL a capacity for input-output operations, data handling, and program length which we have not been able to exhaust during a year of operation. For many situations our approach of adding a very modest "roller" to obtain specialized control functions from a general-purpose language may be a much simpler alternative than "reinventing the wheel" (Pilla, 1973). While critical, the input and output control functions are a minor aspect of programs to run experiments. On-line control involves many bookkeeping operations which, it would seem, most general-purpose languages could handle. Once the technical aspects of control operations are established like reflexes in machine language, the experimenter can more readily concentrate on the organization of the tasks using a high-level language. Interpreter language is particularly valuable in a psychology lab, where research methods are still often exploratory, unforeseen complications requiring further program modifications can develop during the course of a study, and complex experiments may last only a few weeks. While more efficient machine-wise, assembler languages like FORTRAN offer no such ease of program development nor aid to students learning programming, except on larger, more expensive computer systems. For this reason, the FIDO approach may prove worthwhile for on-line control with microcomputers where inexpensive memory now permits trading off computer efficiency in favor of experimenter efficiency.

FIDO enables FOCAL operation of the $1 / 0$ bus, clock, and timed relay settings to be contingent on sense inputs to a PDP-12 computer with at least $8 \mathrm{~K}$ of memory. Since both program space and operating time are major constraints in interactive languages during online control, FIDO manages buffer storage in high memory fields to avoid waiting on tape operations when a number of parameters have to be accessed or stored. FIDO also permits FOCAL to directly test sense 
lines and switches and to go to other assembly language routines in high memory fields. It is possible from FOCAL to reprogram certain features of FIDO such as the clock rate, sense synchronization condition, and addressed memory fields by using a general-purpose PDP command function in FIDO. To minimally interfere FOCAL programming space, FIDO resides mainly on one page in the field 1 display buffer following the suggestion made by Hatcher (1975). Twenty-eight locations in the bottom of the FOCAL user area serve as a gateway to and from FIDO. An explanation of just what FIDO can do is perhaps best given by some examples of how it is used. (Further details on use and a listing are available from the author.)

All FIDO operations are called from within FOCAL with the user function "FX." FX has three arguments which specify the operation and how it is performed. These arguments are conveyed from and to FOCAL using its INTEGER, PUSHJ, and EVAL internal subroutines (Wrege, 0000). To use FIDO from within FOCAL:

$$
\text { Set } \mathrm{X}=\mathrm{FX}(\mathrm{ARG} 1, \mathrm{ARG} 2, \mathrm{ARG} 3)
$$

ARG1 specifies the operation, while ARG2 and ARG3 determine the parameters. The function returns to FOCAL, with $\mathrm{X}$ equal to the value resulting from that operation.

\section{I/O BUS AND PDP COMMANDS}

If $\mathrm{ARG} 1=-1$, a PDP command specified by the octal equivalent of ARG3 is performed with ARG2 in the computer's accumulator. Execution awaits a reprogrammable sense condition which is specified in FIDO. This command can be used to read or output data on the I/O bus. For example:

$$
\text { Set } X=F X(-1,9,3299)
$$

This performs I0T 6343 (see the PDP-12 System Reference Manual) when the appropriate sense line condition is fulfilled. If device 34 is a general-purpose $I / 0$ module such as an M735 (see the Digital Logic Handbook), the function loads Bits 8 and 11 into its output register. Setting ARG3 equal to an instruction to place the contents of the accumulator into a memory location provides a means of reprogramming FIDO from within FOCAL.

\section{CLOCK}

When ARG1 $=-2$, the FX function measures an elapsed time period starting when a sense condition specified by ARG3 is fulfilled and stopping on an above-threshold input to the KW12 clock trigger channel specified by ARG2. For example:

$$
\text { Set } Z=F X(-2,76,1) \text {; Type } Z
$$

These commands wait for sense line 1 to be positive, then start the clock; the clock runs until a trigger input to channel 2; the duration of the elapsed time in milliseconds is typed. The clock rate can be changed from within FOCAL using an FX-1 command.

\section{SENSE FUNCTIONS}

If $\mathrm{ARG1}=-3$, one of two types of tests is performed on the sense switches or sense lines. If $A R G 2=0$, the sense switch or line specified by ARG3 is tested. The function returns to FOCAL with a value of 0 if sense was not set or a value of 4095 if it was set. If ARG2 is greater than zero, FX-3 tests as many senses as specified by ARG2, starting with the switch or line specified by ARG3. The function returns to FOCAL with the decimal equivalent of the first sense found set. If none was set, it returns with a value equal to ARG2 + ARG3. For example:

$$
\text { Set } \mathrm{A}=\mathrm{FX}(-3,3,3) ; \text { Type A }
$$

This command checks sense lines 3,4 , and 5 in succession. If line 4 was positive, A equals 4 , and line 5 is not checked. If none of these is positive, A equals 6 .

\section{ON-LINE FILES AND PROGRAMS}

If $\mathrm{ARG} 1=-4$, FIDO reads the contents of the memory field address apecified by ARG3. Since the contents are added to the value of ARG2, ARG2 is normally set equal to zero. For example:

$$
\text { Set A = FX }(-4,0,2816) \text {; Type A }
$$

FOCAL types the decimal equivalent of the contents of address 5400. If ARG1 $=-5$, FIDO writes the value of ARG2 into the address specified by ARG3. As a check, FIDO subsequently reads the contents of that same address and returns to FIDO with its value. If ARGI $=-6$, FIDO causes FOCAL to go to a machine program stored in some memory field at a starting address specified by ARG3. The value of ARG2 is carried along in the accumulator. To return to FOCAL afterward, it is only necessary for that program to change the data and instruction fields back to 0 and do an indirect jump to address 5102 . The FX-6 function returns with the decimal value of the accumulator's contents at the end of the machine program. The memory field which is read, written, or jumped to can be changed using the FX-1 command.

\section{TIMED OUTPUT}

If $\mathrm{ARG1}=0$ or some positive number, one of two 
operations is performed with the PDP-12 relays. If ARG2 $=0$, the relays are simply set according to the octal equivalent of ARG1. For example:

$$
\text { Set } \mathrm{W}=\mathrm{FX}(33,0,0)
$$

This sets relays 0 and 5 . If ARG2 $>0$, FIDO sets the relays specified by ARG1 for a duration specified by ARG2 starting when sense condition specified by ARG3 is set. Any relays already set are not affected unless again specified in ARG1. All relays in ARG1 are opened after the specified duration. The function returns to FOCAL with the value of the relays which remain set. For example:

$$
\text { Set } W=F X(16,25,52) ; \text { Type } W
$$

This sets relay 1 for $25 \mathrm{msec}$ upon closure of sense switch 4 ; relays 0 and 5 from above remain closed. When completed, the value of $W$ is typed as 33 , which represents the decimal equivalent of the octal code for relays 0 and 5 . To obtain accurate durations of output signals in the millisecond range, we have tapped the relay buffer directly to drive a pair of 7404 hex inverters giving well buffered TTL output signals. For computers other than a PDP-12, precisely timed outputs can be achieved by performing similar operations on a generalpurpose $\mathrm{I} / \mathrm{O}$ module.

Persons attempting to develop similar patches in assembler language for addition to other FOCALs or BASIC might find the following suggestions helpful: (1) Make control of the experiment a two-way street by enabling computer operations to be contingent on other instrumentation, especially when timing is critical. (2) Instead of trying to anticipate all operation parameters, allow the patch to be reprogrammable from with-in the main language. (3) Incorporate a function which can perform any computer command given the proper coding. As well as altering memory locations to achieve Suggestion 2, such a function can permit adding new devices or trying novel operations. (4) Online files can be valuable for quickly selecting particular parameters from larger sets of possible parameters and storing data while the experiment is in progress. Placed in higher memory fields, these files need not interfere with the operating language or program. While such files may be of little interest to owners of disk mem. ories, extra memory may be a much cheaper alternative to those without disks.
Our approach lacks the ability to simultaneously handle more than one experiment or several ongoing real-time functions such as stimulation and data analysis within one experiment. While technically feasible using interrupt levels (Reece, 1973) and careful chaining of operations, multitask programming has not seemed worth attempting on a small computer with little technical support. With the advent of inexpensive microcomputers it may be cheaper to have satellite computers for each overlapping real-time operation on a single complex experiment or to manage several identical experiments simultaneously rather than trying to achieve multiuser real-time sharing on larger computers. Having contingency functions such as present in FIDO would be a simple way to keep the operations of several microcomputers or other instruments synchronized on the same experiment. On-line files may permit other economies by enabling several small computers to share expensive peripherals such as Teletypes and tape decks. Having a main easy-to-program computer first set up and later make on-line calls for analog instrument or microcomputer tasks is an extension of the FIDO approach outside the computer which we have begun to pursue.

\section{REFERENCES}

Castellon, N. J., JR. Standards: Real-time extensions to program systems. Behavior Research Methods \& Instrumentation, 1976, 8, 207-210.

Hatcher, W. E. How to patch FOCAL-12. Decusope, 1975, 14, No.2, 26.

KAYE, M. Overlays to FOCAL-12. DECUS Program Library No. 24. Maynard, Mass: Digital Equipment Users Society, 1971.

Pilla, M. A. Co-existing with on-line systems. Behavior Research Methods \& Instrumentation, 1973, 5, 80-82.

REece, P. Some simple I/0 patches for 4K FOCAL. Decuscope, 1973, 12, No.2, 23-27.

SIDowsKI, J. B. On-line instrumentation in psychology: Dildo or the real thing? Behavior Research Methods \& Instrumentation, 1976, 8, 52-56.

SIEGEL, W. Combining FOCAL and assembly language. Behavior Research Methods \& Instrumentation, 1972, 4, 105-106.

UTTAL, W. R. "Basic black" in computer interfaces for psychological research. Behavior Research Methods \& Instrumentation, $1969,1,35-40$.

WREGE, D. FOCAL: How to write now subroutines and use internal routines. DECUS Publication No. 8-17. Maynard, Mass: Digital Equipment Computer Users Society.

Digital Equipment Corporation. Digital logic handbook. Maynard, Mass: 1973-1977.

Digital Equipment CoRporation. PDP-12 system reference manual. Maynard, Mass: 1970. 